\title{
Efficient Linear Transmission of Complex Waveforms at 216 GHz Using Nonlinear Multiplier Chains
}

\author{
Ali Darwish ${ }^{1}$, Joe Qiu ${ }^{1}$, Edward Viveiros ${ }^{1}$, H. Alfred Hung ${ }^{1}$, and Jeffrey Hesler ${ }^{2}$ \\ ${ }^{1}$ Army Research Laboratory, Adelphi, MD 20783 \\ ${ }^{2}$ University of Virginia, Charlottesville, VA, 22902
}

\begin{abstract}
A solid-state 216-GHz communication link is presented. It utilizes a novel mixer-less transmitter technique to achieve efficient complex modulation using frequency multipliers (which are inherently nonlinear). The transmitter operates with coherent power combining of two frequency multiplier chains to achieve precise linear amplitude and phase modulation despite the strong nonlinearity of the multipliers and the saturated power amplifiers. A 16-QAM modulation signal is demonstrated with a transmitted output power of $100 \mathrm{~mW}$.
\end{abstract}

\section{INTRODUCTION}

$\mathrm{T}_{\mathrm{s}, \mathrm{h}}^{\mathrm{h}}$ he interests in millimeter-wave $(\mathrm{mmW})$ and $\mathrm{THz}$ semiconductor technologies are witnessing very rapid development [1]-[3]. In addition, interest has been growing in using high-mmW and $\mathrm{THz}$ frequencies for communications [4] - [7] and imaging [8]. Power combining has been utilized to obtain significant output power [9]-[10]. Operating frequencies in the $0.2-1 \mathrm{THz}$ range often exceed the cutoff $\left(f_{\mathrm{t}}\right)$ and the maximum frequency of oscillation $\left(f_{\max }\right)$ of most semiconductor devices. As such, the availability of many active components (such as low-noise amplifiers, power amplifiers, and active mixers) is limited, and one is left with passive components. As a result, the attainable solid-state output power available at this frequency range is limited. Many transmitters have relied on the use of frequency multipliers and/or passive mixers to generate and modulate signals. Most of these blocks are either intrinsically nonlinear (e.g. frequency multipliers) or are operated nonlinearly (e.g. saturated amplifiers) to obtain the highest power. Thus, most of the communication links demonstrated in this frequency regime, so far, have relied on simple modulations such as on-off keying (OOK), or low order phase modulations such as binary phase shift keying (BPSK), quadrature phase shift keying (QPSK). However, the spectral efficiency of such modulation schemes is limited compared to higher order modulations (such as 32QAM) that have varying-amplitudes. Reports of higher order modulations are very few [6], and [7] (where a $670 \mathrm{GHz}$ 64QAM link utilizing InP devices was demonstrated); and they usually require some output power back-off. Supporting communications links with varying-amplitude modulations at the high frequency regime introduces several problems. First, power generation and amplification are difficult. Second, there are strong nonlinearities present, in most cases, caused by the use of multipliers and the desire to obtain maximum power from amplifiers and mixers. Third, setting up a frequency plan to eliminate unwanted signals (such as image rejection) requires low-loss high-order filters that are difficult to realize. Thus, many of the demonstrations are done with double-side band signals.

This paper presents a novel mixer-less technique for generating general-purpose modulations (such as nQAM with varying amplitude/phase) in the presence of highly nonlinear components (such as frequency multipliers, and amplifiers operating in highly saturated mode). The new transmitter architecture is termed direct quadrature transmitter (DQtransmitter). It was inspired by the linear amplification using nonlinear components (LINC) architecture technique [11]-[12], and our earlier work on continuous phase modulation (CPM) where full power (no back off) was used with a 16-level signal [13]. To the best of our knowledge, this is the first demonstration of a variable amplitude/phase 16QAM modulation at frequencies above $200 \mathrm{GHz}$ with significant output power. The single side band signal is obtained without the use of filters. The improvement in output power is the result of avoiding the use of mixers (which have limited convergence efficiency) and filters. Additionally, there is no need to backoff on power since the architecture works efficiently even with strong nonlinearities in the transmit chain.

\section{DQ-TRANSMITTER ARCHITECTURE}

The operation of the DQ-Transmitter relies on a simple concept. Consider Fig. 1 where two equal-amplitude vectors, $(=|\underline{\mathbf{a}}| \angle \Phi \mathrm{a})$, and $\underline{\mathbf{b}}(=|\underline{\mathbf{b}}| \angle \Phi \mathrm{b})$, are added to produce their vectorsum $\underline{\mathbf{C}}(=|\underline{\mathbf{C}}| \angle \Phi \mathrm{c})$. By adjusting the angles $\Phi \mathrm{a}$, and $\Phi \mathrm{b}$, one can produce a vector $\underline{\mathbf{C}}$ with any arbitrary angle $\Phi \mathrm{c}$, and any arbitrary amplitude $|\underline{\mathbf{C}}|$; provided that $|\underline{\mathbf{C}}| \leq|\underline{\mathbf{a}}|+|\underline{\mathbf{b}}|=2|\underline{\mathbf{a}}|$.

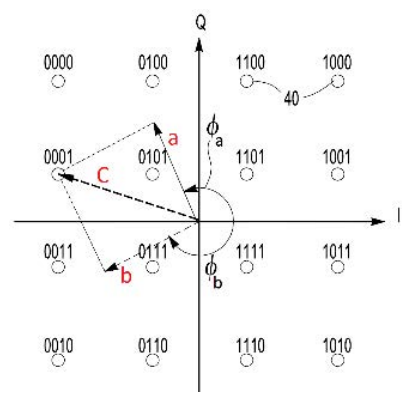

Fig. 1 Example of construction of an arbitrary vector $\mathrm{C}(=|\mathrm{C}| \angle \Phi \mathrm{c})$ using two equal amplitude vectors $\mathrm{a}(=|\mathrm{a}| \angle \Phi \mathrm{a})$, and $\mathrm{b}(=|\mathrm{b}| \angle \Phi \mathrm{b})$. 


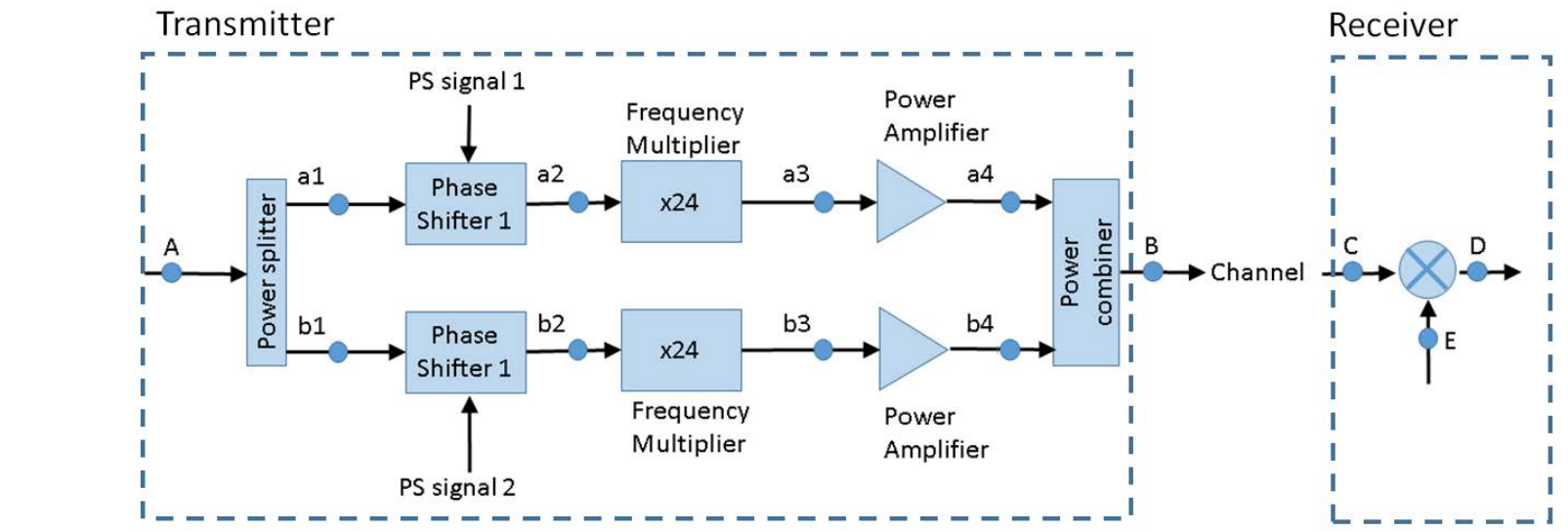

Fig. 2 Schematic of DQ-Transmitter and receiver.

A schematic of the DQ-Transmitter is shown in Fig. 2. It operates as follows. A single-tone source signal, say at $12 \mathrm{GHz}$, enters at point A where it gets equally split (into $\underline{\mathbf{a} \mathbf{1}}$, and $\underline{\mathbf{b 1}}$ ) and forwarded to a pair of phase shifters. After that, each signal gets frequency multiplied by $\times 18$, say, and amplified using amplifiers which can operate at high power saturation. If the components in the upper and lower branches are identical, then the signals at $\underline{\mathbf{a} 4}$, and $\underline{\mathbf{b} 4}$ will be equal in amplitude. If they are not, then variable attenuators (not shown) can be used to equalize the two constant-amplitude signals. Once the signal amplitudes at a4, and b4, are equal, they are then combined coherently to form the transmit signal. Phase controls 1, and 2, at PS signal 1 and 2, respectively, allow one to set the phase and amplitude of the transmit signal to any value; when the vectors add in phase, maximum amplitude is achieved, and when they add out of phase, they cancel completely.

In Fig. 2, the phase shift is set before the frequency multiplication. Hence, the phase shift gets multiplied similar to the frequency. For example, if one would like to set $\Phi \mathrm{a}=90^{\circ}$, at point a 4 , then phase shifter 1 is set to $5^{\circ}$ since $5^{\circ} \times 18=90^{\circ}$, and so forth. The location of the phase shifter along the transmitter chain could have been chosen after the frequency multiplication (i.e. after the generation of the $216 \mathrm{GHz}$ signal). However, it is more cost-effective, and lower in signal loss, to implement phase shifting at lower frequencies, around $12 \mathrm{GHz}$, compared to that at $216 \mathrm{GHz}$. Additionally, any signal attenuation at $12 \mathrm{GHz}$, caused by the phase shifter, can be easily corrected with addition of gain; this is not true at $216 \mathrm{GHz}$ where gain and power are at a premium.

\section{DQ-TRANSMITTER EXPERIMENTAL RESULT}

This section presents the experimental results of implementing the DQ-transmitter. A picture of the DQtransmitter experimental setup is shown in Fig. 3. A frequency synthesizer source is used to supply a $12 \mathrm{GHz}$ continuous wave $(\mathrm{CW})$ tone which is power divided into two equal signals. After that, a $12 \mathrm{GHz}$ analog phase shifter is used to set the phase of each signal. The $12 \mathrm{GHz}$ signal is then frequency multiplied by $\times 2$, and $\times 3$ passive Virginia Diode Inc. (VDI) parts. The 72 $\mathrm{GHz}(=12 \mathrm{GHz} \times 6)$ signal is fed to a Northrop Grumman
Aerospace Systems (NGAS) tripler/amplifier which generates a $216 \mathrm{GHz}$ amplified signal. The amplifier has a saturated output power of $60 \mathrm{~mW}$. The two branches are then combined using a hybrid coupler to generate around $100 \mathrm{~mW}$. The signal is radiated using a horn antenna with $20 \mathrm{dBi}$ of gain. The receiver is composed of a horn antenna, with $20 \mathrm{dBi}$ of gain, feeding a VDI subharmonic mixer with a local oscillator (LO) with frequency equal to $110 \mathrm{GHz}$. The IF frequency, around 4 $\mathrm{GHz}$, is fed to a low noise amplifier (LNA), and received using a standard receiver. The LO is generated by tripling a 36.67 $\mathrm{GHz}$ using a VDI multiplier.

The output of the receiver in Fig. 3 is connected to an oscilloscope showing a square-wave amplitude pattern. It is the result of a square wave modulation of the phase of one of the multiplier chains by $180^{\circ}$. The ratio of power during ON/OFF periods is over 100, which indicates effective addition/cancellation of the vectors.

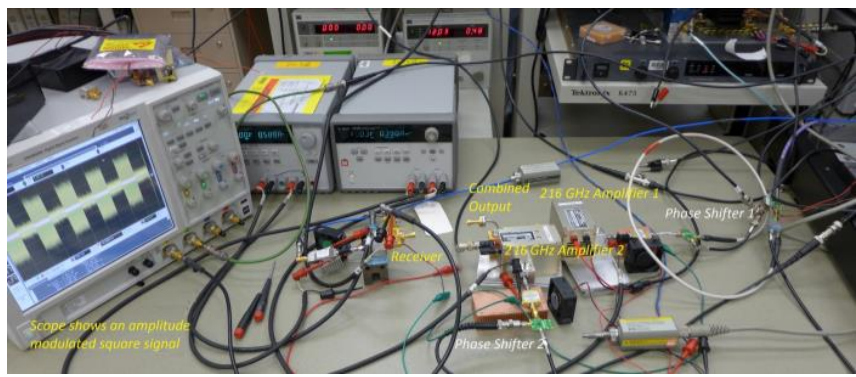

Fig. 3. Picture of DQ-transmitter setup.

To verify the operation of the system, a simple BPSK modulation was generated by modulating both phase shifters with a $10^{\circ}$ (since $\left.10^{\circ} \times 18=180^{\circ}\right)$ square wave. The result is shown in Fig. 4. One can clearly see in the IQ constellation, the two discrete states. This case shows the phase modulation capability of the system. Excellent signal quality was observed. The Figure also shows the receiver spectrum. 


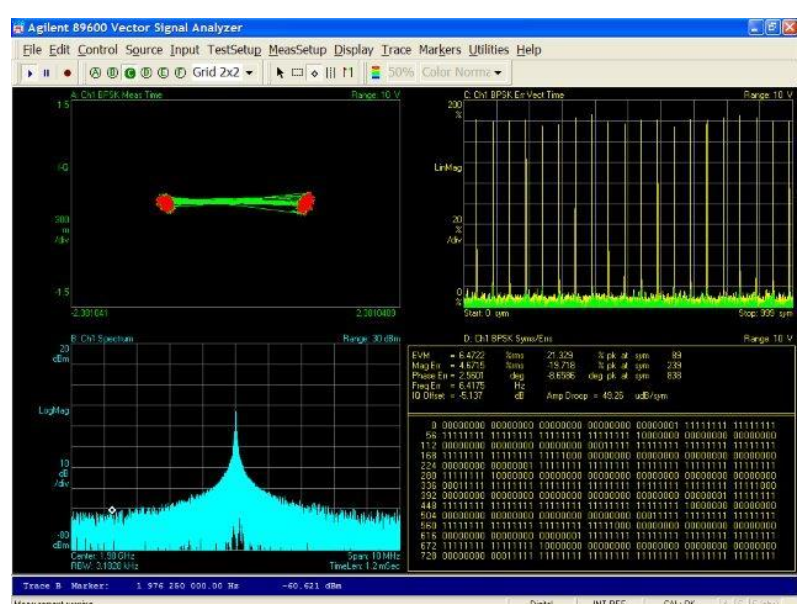

Fig. 4. Screen capture of vector signal analyzer showing received BPSK signal.

To test the system's ability to control phase and amplitude simultaneously, a 16-QAM signal was encoded and transmitted with a symbol rate $(1 / \mathrm{T})$ of $5 \mathrm{Msymbol} / \mathrm{s}$ which is equivalent to 20 Mbit/s. Fig. 5 shows the received signal. The I-Q constellation shows 16 distinct states, as expected with excellent signal quality. This result is interesting as it proves the ability of the transmitter to modulate the phase and amplitude simultaneously, despite the fact that both signal chains are very nonlinear (containing frequency multipliers and a highly saturated amplifier). The Figure also shows the spectrum of the received signal. An error vector magnitude (EVM) of $5.7 \%$ is obtained without any system calibration or error correction. One can produce and receive more complex digital modulation signals by programming the phase shifters appropriately.

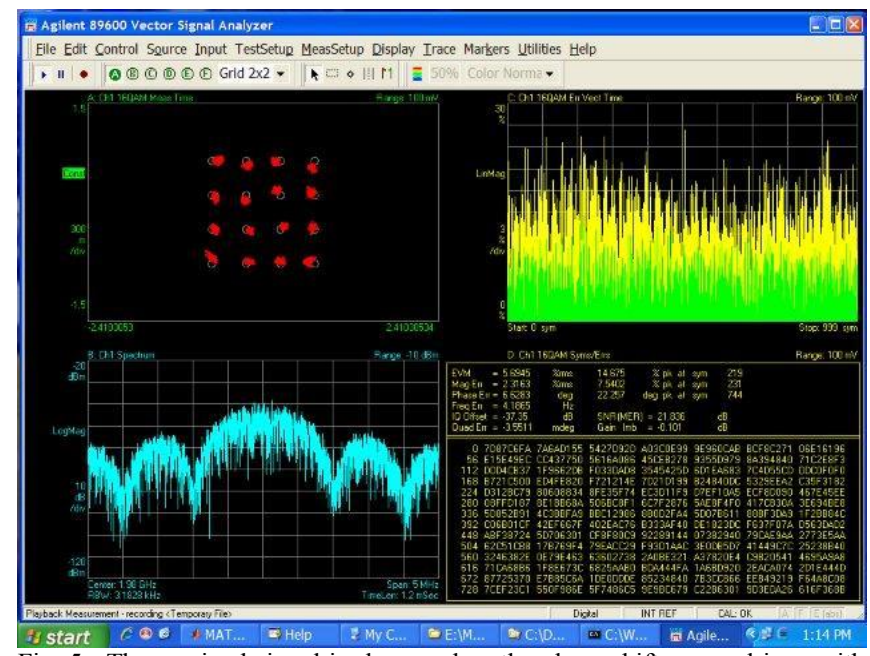

Fig. 5. The received signal is shown when the phase shifters are driven with 16-state, quadrature amplitude modulation (16-QAM) signals. One can clearly see 16 discrete states in the IQ constellation with excellent signal quality.

The DQ-transmitter has several key advantages over the typical mixer-based transmitter where the IF is up-converted to $\mathrm{mmW}$ using a LO. Once power at the high frequency signal (216 GHz, say) is generated, it can be transmitted without attenuation (from the mixer's convergence efficiency). This is a significant advantage since generating high power at $216 \mathrm{GHz}$ is difficult. Generally, the conversion efficiency of mixers at high frequency is very limited, requiring high frequency power amplifiers to increase $\mathrm{mmW}$ signal (to offset mixer losses); currently available mmW amplifiers have poor linearity and can severely degrade the signals they amplify. Last, in the mixer case, obtaining a single side band signal requires filters, which add loss.

\section{CONCLUSION}

A general-purpose efficient $216 \mathrm{GHz}$ transmitter approach has been presented. It supports varying-amplitude and phase modulations, as well as analog modulations. The new architecture was demonstrated with a single side band 16-QAM transmit signal at $100 \mathrm{~mW}$ level in the communication link. The approach directs almost all the $\mathrm{mmW}$ power generated towards the transmitted signal resulting in much higher power than the conventional mixer-based transmitter architectures. The technique also allows the control of the signal trajectory between symbols. For example, a trajectory that does not pass through zero can be selected in order to improve the spectral efficiency.

\section{ACHKNOWLEDGMENT}

The authors would like to acknowledge NGAS, and Dev Palmer of DARPA for their support.

\section{REFERENCES}

[1] H.B. Wallance, "DARPA MMW System Programs and How They Drive Compound Semiconductor Technology Needs," IEEE Compound Semiconductor IC Symp. Dig., Oct 2015.

[2] W. R. Deal, K. Leong, A. Zamora, V. Radisic, and X.B. Mei, "Recent progress in scaling InP HEMT TMIC technology to $850 \mathrm{GHz}$," IEEE MTT-S International Microwave Symposium, Tampa, FL, June 2014.

[3] J. D. Albrecht, M.J. Rosker, H.B. Wallace, T.-H. Chang, "THz electronics projects at DARPA: Transistors, TMICs, and amplifiers," IEEE MTT-S International Microwave Symposium, pp. 1118-1121, Anaheim, CA, May 2010.

[4] Ho-Jin Song, T. Nagatsuma, "Present and Future of Terahertz Communications," IEEE Transactions on Terahertz Science and Technology, vol. 1, issue 1, pp. $256-263,2011$

[5] Weiwen Yuan, Hanqing Wang, Huiyuan Li, Zhuo Zhang, Xiaojie Yang, and Weixun Shi, "0.3THz wireless communication systems for space applications,” Proc. SPIE 9795, Selected Papers of the Photo-electronic Technology Committee Conferences, July 2015.

[6] I. Kallfass et al., "All active MMIC-based wireless communication at 220 GHz,” IEEE Trans. THz Sci. Tech., vol. 1, no. 2, pp. 477-487, Nov. 2011

[7] B. Deal, K. Leong, " $850 \mathrm{GHz}$ wireless link demonstration using InP HEMT," 2015 IEEE International Microwave Symposium, Terahertzwave Wireless Communications Workshop, Phoenix, AZ, 17-22 May, 2015.

[8] UR Pfeiffer, Y Zhao, J Grzyb, R Al Hadi, “A 0.53THz reconfigurable source array with up to $1 \mathrm{~mW}$ radiated power for terahertz imaging applications in $0.13 \mu \mathrm{m}$ SiGe BiCMOS,” 2014 IEEE International SolidState Circuits Conference Digest of Technical Papers (ISSCC), pp. $256-$ 257, San Francisco, CA, 2014.

[9] Z. Griffith, M. Urteaga, P. Rowell, R. Pierson, "A $23.2 \mathrm{dBm}$ at $210 \mathrm{GHz}$ to $21.0 \mathrm{dBm}$ at $235 \mathrm{GHz} 16$-way PA-cell combined InP HBT SSPA MMIC", IEEE Compound Semiconductor IC Symp. Dig., Oct 2014.

[10] D. Gritters, K. Brown, E. Ko, Z. Griffith, M. Urteaga, "200-260 GHz Solid-State Amplifier with $700 \mathrm{~mW}$ of Output power," IEEE Interational Microwave Symp. Dig, May 2015.

[11] D. C. Cox, "Linear amplification with nonlinear components," IEEE Transactions on Communications, 1974, vol. 23, pp. 1942-1945.

[12] D. C. Cox and R. P. Leck, "Component signal separation and recombination for linear amplification with nonlinear components," IEEE Transactions on Communications, 1975, vol. 23, pp. 1281-1287.

[13] Joe Qiu, C. Dietlein, T. Ivanov, D. Wikner, A. Hedden, E. Viverios, H. A Hung, "Efficient G-band digital communications using continuous phase modulation,” IEEE Int'1 Microwave Symp., Montreal, Canada, 2012. 